\title{
The Physics Programme of MICE Step IV
}

\author{
Ryan Bayes*广 \\ University of Glasgow \\ E-mail: ryan.bayeseglasgow.ac.uk
}

The International Muon Ionization Cooling Experiment is progressing towards a full demonstration of the feasibility of ionization cooling technology decisive for muon colliders. Step IV provide the first precise measurements of emittances. The components required for Step IV, including spectrometer solenoids, muon trackers and absorber-FC (focus coil) modules have been assembled with data collection in 2015. The physics programme of this Step will be described in detail.

16th International Workshop on Neutrino Factories and Future Neutrino Beam Facilities - NUFACT2014, 25 -30 August, 2014

University of Glasgow, United Kingdom

* Speaker.

${ }^{\dagger}$ MICE Collaboration 


\section{MICE Background}

Neutrino Factories are the pre-eminent future facilities for the ongoing study of neutrino physics. A Neutrino Factory uses the decay of muons in a storage ring to produce neutrino beams with precisely known composition and energy spectra [1]. As part of the acceleration chain the muon beam must be cooled transverse to the direction of travel. Cooling reduces the physical size of the beam as well as the transverse component of the muon momentum. Cooling the beam increases the number of muons in the beam that is accepted by the accelerator channel and the ultimate performance of the experimental facility [2]. Because of the half life of muons, conventional cooling methods are not effective. The Muon Ionization Cooling Experiment (MICE) was proposed to provide a seminal demonstration of cooling through ionization energy loss which is the only known method of reducing the muon beam phase space on the time scale required for a Neutrino Factory.

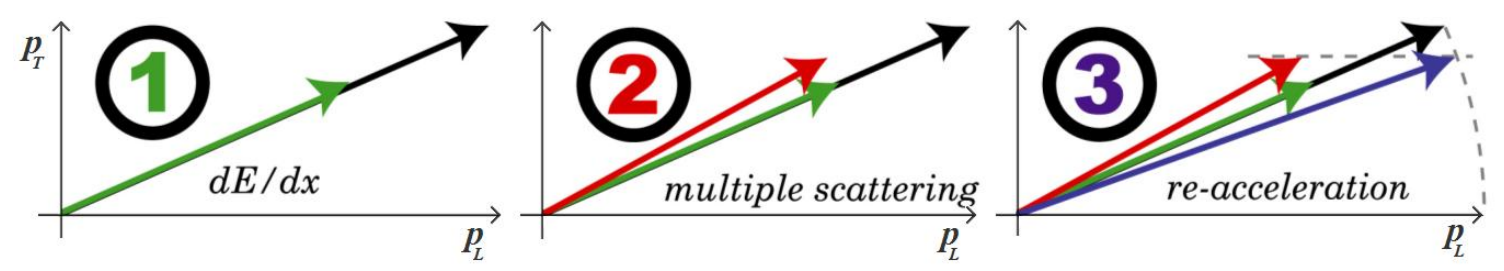

Figure 1: An illustration of ionization cooling.

Ionization cooling works through sequential reduction of momenta and re-acceleration of muons along the beam line. This change in the momentum of the beam is shown graphically in Fig. 1. Particles are transported into the absorber material with an initial momentum vector (shown in blue). The energy loss acts to reduce the momentum of the particles to the vector shown in green. However the multiple Coulomb scattering of the particles in the absorber introduces a random component to the momentum that can increase the transverse component of the particle momentum. Re-acceleration in the beam direction then produces net cooling in the transverse direction. The effect of cooling is measured using the multi-dimensional variance of the muon position and momentum distributions, known as the emittance, $\varepsilon_{n}^{4 D}=\sqrt[4]{\left|V^{4 D}\right|} / m$ [4]. The change in the emittance for a beam passing through a material can be described by the cooling equation

$$
\frac{d \varepsilon}{d s}=\frac{-\varepsilon_{n}}{\beta^{2} E}\left\langle\frac{d E}{d X}\right\rangle+\frac{\beta_{t}(13.6 \mathrm{MeV})^{2}}{2 \beta^{3} E m_{\mu} X_{0}} .
$$

The change in the emittance can be separated into a cooling contribution, primarily dependent on the mean energy loss in the scattering material, and multiple scattering contribution which depends on the transverse betatron function $\beta_{t}$, which describes the oscillations of the beam in phase space and inversely on the mean free path of the muon in the scattering material, $X_{0}$. Both terms also depend on the beam energy, $E$ and the relativistic boost $\beta$. The equilibrium beam emittance is reached when

$$
\varepsilon_{n}=\frac{\beta_{t}(13.6 \mathrm{MeV})^{2}}{2 \beta m_{\mu} X_{0}}\left\langle\frac{d E}{d X}\right\rangle^{-1}
$$


so it is important to test low $\mathrm{Z}$ materials to maximize both the mean free path and the mean energy loss.

The measurement of the properties of such materials, specifically liquid hydrogen and lithium hydride, will be important milestones in MICE. Sustained transverse beam cooling can only be achieved if re-acceleration is applied in the longitudinal direction. However, such a measurement cannot be completed if the dependence of the cooling effect on the material properties are not known. Thus the measurement of material dependences is the primary motivation of the current stage in MICE, Step IV.

\section{MICE Step IV in Detail}

MICE Step IV is nearing completion as of this writing. The experiment is located at the Rutherford Appleton Laboratory (RAL) and uses a beam line parasitic to the ISIS synchrotron to provide the muons required for the cooling studies. The apparatus is built around a central absorber with independent measurements of the particle momentum and identification upstream and downstream of the absorber. The absorber is placed in the centre of an assembly that contains two superconducting coils whose purpose is controlling the transverse size of the beam as it passes through the absorber. Beyond the focus coils are two superconducting coils to contain the scintillating fibre trackers, each with two end coils to shape the magnetic fields entering and exiting the spectrometer solenoid, and two match coils to connect the spectrometer and focus coil fields. A schematic of MICE Step IV appears in Fig. 2 while a photograph of the experiment as built is shown in Fig. 3.

The measurement of the emittance and material properties are primarily conducted using the scintillating fibre trackers. These devices consist of five stations each composed of three planes, each oriented $60^{\circ}$ with respect to the others. Each tracker is 1.19 metres long with the planes at irregular intervals selected to reduce degeneracies in the reconstruction of trajectories. The tracker volume is filled with Helium gas, while the volume between the trackers and the absorbers is evacuated. The trackers have a position resolution of $470 \mu \mathrm{m}$ and a momentum resolution $\Delta p / p \approx 1 \%$.
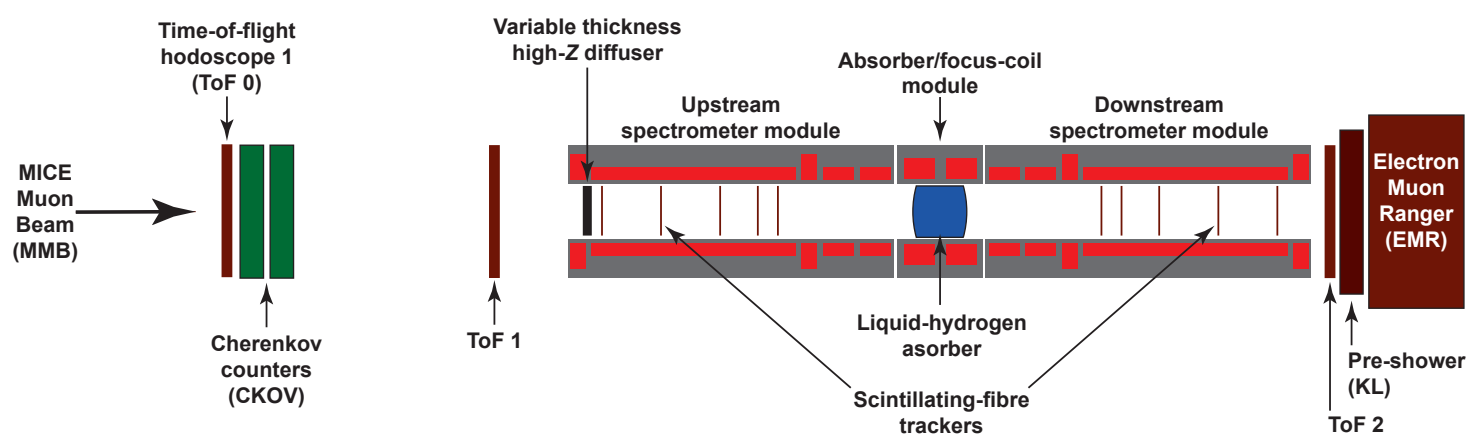

Figure 2: A labelled schematic of the Step IV MICE cooling channel. 


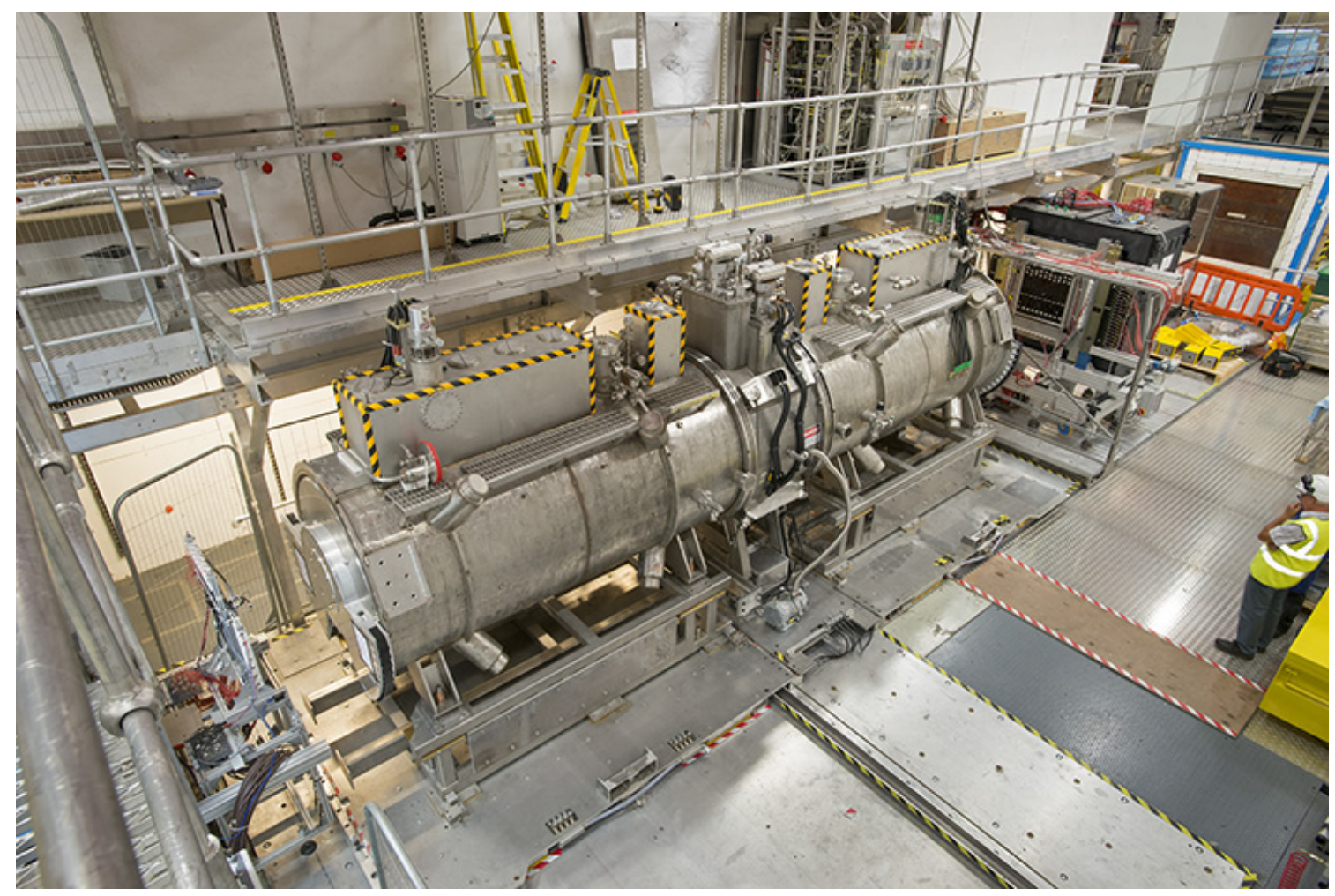

Figure 3: The cooling channel as it now exists in the MICE Hall at RAL.

The emittance measurements require a muon sample, with contamination from pions less than $0.1 \%$, to achieve the required accuracy. A redundant suite of detectors are used in MICE to provide this degree of particle identification. A set of three time of flight (TOF) detectors; one at each end of the cooling channel, and one $11 \mathrm{~m}$ upstream of the absorber as part of the muon beam line; provide the first indication of the particle identity, as electrons, muons, and pions have very different transit times at equal momenta. A Cherenkov detector, at the same position as the far upstream time of flight detector, also provides a velocity based separation of muons, electrons, and pions in the beam. A pre-shower detector, called the KLOE Light or KL detector, immediately behind the TOF detector downstream of the cooling channel, provides separation between minimum ionizing particles and particles that produce electro-magnetic or hadron showers. The last detector consists of a scintillating detector with a cubic metre of active volume composed of 2832 scintillating bars with a triangular cross-section organized in 48 alternating $\mathrm{x}$ and y planes and provides a measurement of particle energy from the range, hence its name; the Electron-Muon Ranger (EMR) .

The experiment will test the transport of muons through the absorber at various settings to establish behaviours associated with different physical processes. A matrix showing the proposed settings is shown in Table 1. The initial beam emittance is manipulated through the use of a diffuser assembly that introduces material into the beam to inflate its emittance prior to traversing the cooling channel. The diffuser contains four planes, two of brass, with $2.6 \mathrm{~mm}$ and $5.6 \mathrm{~mm}$ thicknesses, and two of tungsten with the same thicknesses, which may be moved independently allowing for 16 different settings. The initial momentum is manipulated by matching the conventional MICE 
beam into the super-conducting magnets of the cooling channel.

Not reflected in the matrix is the potential for changes in the absorber material. A liquid hydrogen volume and a lithium hydride disk have been constructed to serve as the absorber material and there are plans to use both in Step IV running, effectively increasing the number of settings by a factor of 2. Plans also exist to take a limited amount of data with negative muons in addition to the positive muons that are to be used by default. A set of data with no solenoid field will also need to be taken for alignment and scattering studies. As a result there are, potentially, 54 different cases that can be considered by MICE Step IV. These studies will be prioritized before data collection starts in 2015.

\begin{tabular}{|c|c|c|}
\hline $\begin{array}{c}3 \pi \\
140 \mathrm{MeV} / \mathrm{c}\end{array}$ & $\begin{array}{c}6 \pi \\
140 \mathrm{MeV} / \mathrm{c}\end{array}$ & $\begin{array}{c}10 \pi \\
140 \mathrm{MeV} / \mathrm{c}\end{array}$ \\
\hline $3 \pi$ & $6 \pi$ & $10 \pi$ \\
$200 \mathrm{MeV} / \mathrm{c}$ & $200 \mathrm{MeV} / \mathrm{c}$ & $200 \mathrm{MeV} / \mathrm{c}$ \\
\hline $3 \pi$ & $6 \pi$ & $10 \pi$ \\
$240 \mathrm{MeV} / \mathrm{c}$ & $240 \mathrm{MeV} / \mathrm{c}$ & $240 \mathrm{MeV} / \mathrm{c}$ \\
\hline
\end{tabular}

Table 1: Matrix of planned beam line and diffuser settings to be used during Step IV running. These nine settings are supplemented by the addition of different absorbers and beam charges with the solenoid fields both on and off, inflating the number of studies significantly.

\section{The Physics of Ionization Cooling}

The physics programme for MICE centres around the components of the cooling equation. The central measurement is that of the emittance of the muon beam and its change across the absorber. Ancillary to this measurement are those of the mean energy loss, the multiple scattering and determination of the background contamination.

The measurement of the emittance in MICE Step IV is currently under study. Preliminary results from simulations of Step IV have been generated which show that the emittance can be measured in the MICE trackers with a precision of $0.01 \mathrm{~mm}$ using $8 \times 10^{5}$ muons. The expected drop in the emittance across the absorber is on the order of $0.3 \mathrm{~mm}$ and the result measured from the simulation is in agreement with the ideal result, after applying corrections for the correlations in the variance. This result is shown in Fig. 4.

Simulation studies of multiple scattering have been considered with particular emphasis on the theoretical modelling of multiple scattering. Multiple scattering is exceptionally important to MICE not only because it is the heating term in the cooling equation but also because is is poorly understood in low $\mathrm{Z}$ materials [5]. This is illustrated in Fig. 5 (left). MICE can address this problem by matching tracks measured in the upstream spectrometer to those measured in the downstream in both field on and field off cases. The results can then be used for input to build new models for multiple scattering at high angles where the largest deviation is observed as illustrated in Fig. 5 (right). A similar measurement can be conducted for the confirmation of the energy loss models. 


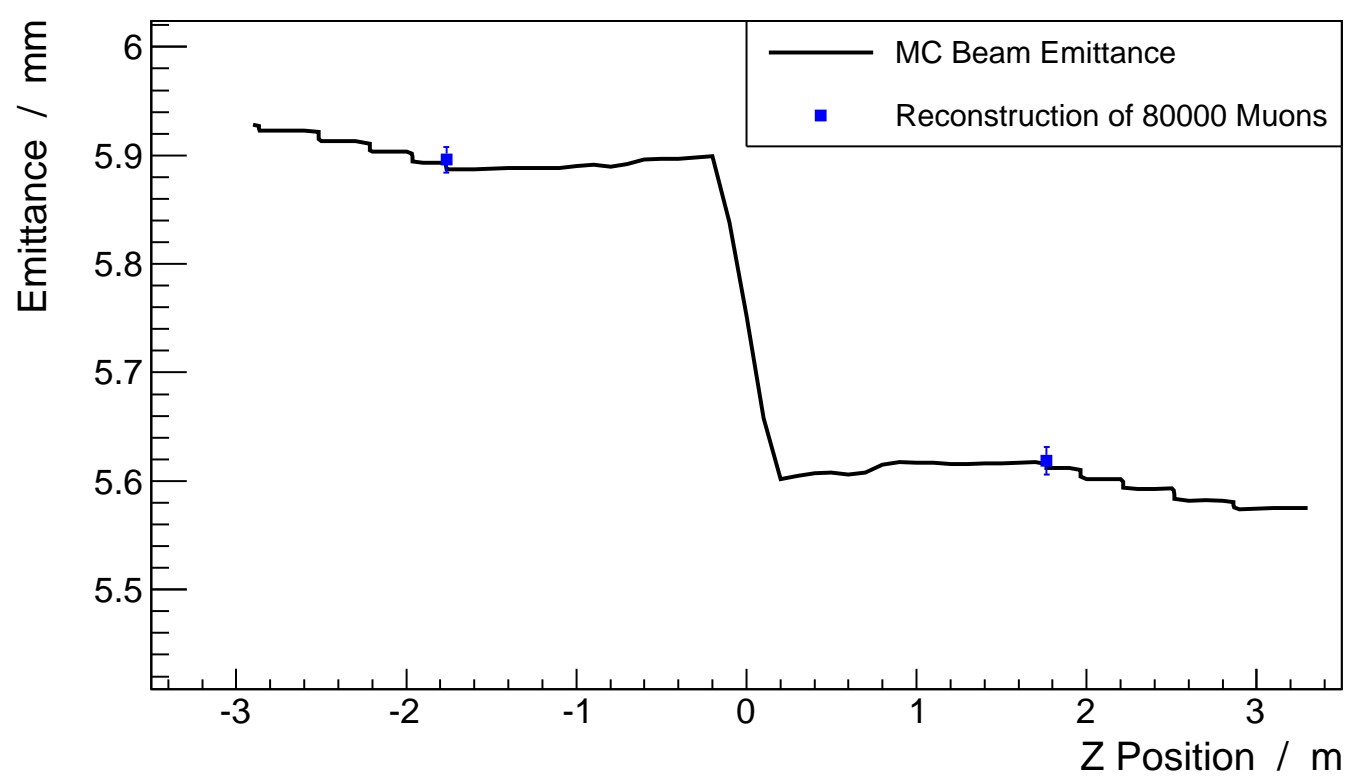

Figure 4: Emittance measurement conducted in a simulation of the MICE step IV cooling channel. The line represents the true value of the emittance determined from the Monte Carlo truth information. The points represent the measurement determined from the tracker reconstruction in the simulation. Figure provided by Chris Hunt of Imperial College London
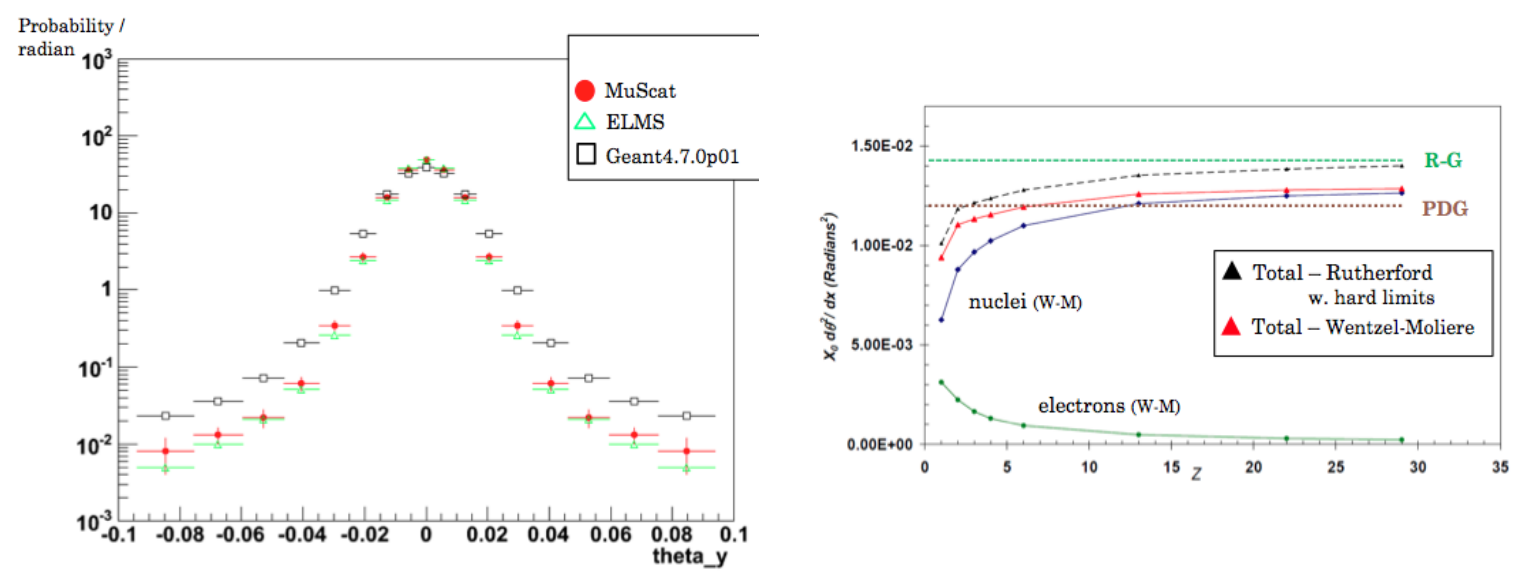

Figure 5: Status of scattering studies in low $\mathrm{Z}$ materials [5]. Left: the scattering width predicted for low $\mathrm{Z}$ materials using various models compared to data. Right: the scattering distribution collected by the MuScat experiment [6] compared to two different scattering models. 


\section{Schedule and Outlook}

As stated before, MICE Step IV is currently under construction, with completion scheduled for Spring of 2015 with commissioning runs to follow. Data collection is now expected to continue through to June of 2016. The end of Step IV is set by a requirement that the construction of the final stage of MICE; the demonstration of ionization cooling; is complete by the end of September 2017. As such the data collection period is limited by the time required to decommission Step IV prior to the construction of the demonstration of ionization cooling.

The preliminary steps of MICE, including commissioning the beam line and particle ID detectors, have been completed. The physics program of MICE Step IV will provide a number of results that will be important for the demonstration of ionization cooling. The most significant of these will be the measurement of emittance reduction by ionization with no acceleration. An integral measurement of the multiple scattering will be conducted in low $\mathrm{Z}$ materials which will be exceedingly useful for the validation of new multiple scattering models. To take full advantage of the opportunities presented by the MICE step IV setup an intensive data collection period will take place during 2015 and 2016.

\section{References}

[1] M. Bogomilov et.al., Phys. Rev. ST Accel. Beams. 17, 121002 (2014).

[2] C.T. Rogers et.al., Phys. Rev. ST Accel. Beams. 16, 054801 (2013).

[3] R. Edgecock, J. Phys. G 291601 (2003)

[4] C.T. Rogers, "Beam Dynamics in an Ionization Cooling Channel", Thesis, Imperial College London (2008)

[5] T. Carlisle, "Step IV of the Muon Ionization Cooling Experiment and the Multiple Scattering of Muons", Thesis, University of Oxford, (2013)

[6] D. Attwood et al., Nuc. Instrum. Meth. B, 251(1) 41 (2006). 